\title{
In silico characterization of three two-component systems of Ehrlichia canis and evaluation of a natural plant-derived inhibitor
}

\author{
E.V. Santos, G. Silva, G.P. Cardozo, T.A. Bitencourt, S.C. França, \\ A.L. Fachin and M. Marins \\ Unidade de Biotecnologia, Universidade de Ribeirão Preto, \\ Ribeirão Preto, SP, Brasil \\ Corresponding author: M. Marins \\ E-mail: marins@heranza.com.br
}

Genet. Mol. Res. 11 (4): 3576-3584 (2012)

Received November 24, 2011

Accepted March 15, 2012

Published October 4, 2012

DOI http://dx.doi.org/10.4238/2012.October.4.5

\begin{abstract}
Two-component signal transduction systems (TCS) are important elements in the interaction of endobacteria with host cells. They are basically composed of two proteins, an environmental signal sensor and a response regulator, which activate genes involved in a wide range of bacterial responses to their environment. We analyzed three sets of genes corresponding to TCS of Ehrlichia canis, a common tick-borne canine pathogen and the etiologic agent of canine monocytic ehrlichiosis, in order to identify the characteristic domains of the sensor and response regulator components. Analysis of sequence alignments of the corresponding proteins indicated a high degree of similarity to other members of the Anaplasmataceae TCS proteins, demonstrating that they could be useful as universal targets for development of new drugs against these bacteria. We also evaluated by quantitative PCR inhibition of $E$. canis by (2H)-1,4-benzoxazin-3(4H)-one (BOA), the core compound of the plant phenolic compound DIMBOA, which shows inhibitory action against TCS of the phytopathogen Agrobacterium tumefasciens. This bacterium exerts its pathogenicity
\end{abstract}


by transferring oncogenic DNA (T-DNA) into plant cells; this transfer is mediated through a type-IV secretion system, which is regulated by the VirA/VirG TCS. The process of infection and pathogenesis of $E$. canis is associated with the secretion of effector proteins into the host cell cytoplasm through a T4SS system, which blocks the cell defense response. We suggest that $\mathrm{BOA}$, and possibly other plant phenolic compounds that are TCS inhibitors, can be exploited in the search for new antiehrlichial drugs to be used alone or as complements in the treatment of canine monocytic ehrlichiosis.

Key words: Ehrlichia canis; Anaplasmataceae; Two-component system

\section{INTRODUCTION}

As cosmopolitan microorganisms, bacteria are in contact with a wide variety of chemical compounds in the environment. Many of these compounds are sources of indispensable nutrients for survival, whereas others are toxic to them. These compounds add to the highly diverse and complex environmental signals to which bacteria are subjected. Their capacity to process and respond to the information driven by these environmental signals is based in the gene toolbox carried by each species that allows it to inhabit, survive, and reproduce in specific and varying environments (Armitage, 1992; Miller et al., 2009). One of the tools carried by most bacterial species studied so far is a group of genes coding proteins that constitute signal transduction two-component systems (TCSs). These genes are usually located within a single operon, and their products form the two basic elements of a TCS, which are a transmembrane sensor protein carrying histidine kinase (HK) and a corresponding cytoplasmic protein known as a response regulator (RR) (Gao and Stock, 2009).

Bacterial HKs are characterized by a field sensor extracytoplasmic domain in the Nterminal region and $\mathrm{H}^{+}$-ATPase activity in the $\mathrm{C}$-terminal region, distinguishing it as a phosphoacceptor domain. The sensor domain is highly variable in size and amino acid sequence identity, ensuring specificity to several environmental signals. Each HK captures a particular type of signal according to the size and amino acid sequence of its field sensor (Laub and Goulian, 2007).

The phosphoacceptor domain is highly conserved, especially within a joint of six subdomains known as $\mathrm{H}, \mathrm{N}, \mathrm{F}, \mathrm{G} 1, \mathrm{G} 2$, and G3. The H subdomain is defined by an invariant histidine residue that is phosphorylated when the sensor domain receives a signal from the environment. The $\mathrm{N}$ subdomain contains an asparagine residue, whereas G1, G2, and G3 contain several glycine residues. The RR is usually a transcription factor and is also composed of two major domains, an N-terminal receiver domain and a DNA-binding domain in the C-terminal region. The receiver domain is conserved among various RRs and has an invariant aspartate residue, whereas the DNA-binding domain is highly variable in its amino acid sequence. This variability is important because different genes are regulated by a TCS according to the sequence specificity of this domain (Lavin et al., 2007).

Signal transduction occurs via phosphotransfer reactions between an HK and its corresponding RR (Figure 1). The HK primarily monitors environmental signals through its sensor domain. The interaction with specific signals promotes autophosphorylation of $\mathrm{HK}$, mediated by the $\mathrm{H}^{+}$-ATPase domain, which transfers a high-energy phosphate from an ATP molecule to the invari- 
ant phosphoacceptor histidine residue domain. The next step of the process is a phosphotransfer reaction involving the transfer of the phosphate from the histidine residue to the invariant aspartate residue present in the receiver domain of a corresponding $R R$. This phosphorylation activates the transcriptional regulatory function of the DNA-binding domain of the RR, which involves binding to regulatory sequences that can induce or repress the transcription of specific genes.

TCSs are involved in the transduction signal of a wide variety of environmental stresses such as nutrient deficiency, heat and cold shock, osmotic alteration, low $\mathrm{pH}$, and others (Stock et al., 2000; Gao and Stock, 2009). Moreover, TCSs are involved in a number of cellular processes such as anaerobic respiration, nitrogen and phosphate acquisition, host cell recognition by the pathogen, cell viability, sporulation, production of chemotoxins, and expression of virulence factors. Also involved in these processes are enzymes that metabolize complex proteins and glycoproteins, bacterial toxins, cell surface proteins and carbohydrates, protective proteins, and hydrolytic enzymes. In the blood cell parasite Brucella abortus, for example, the TCS BvrR/BvrS regulates the process of host cell invasion and survival of the pathogen within the host by regulating the formation of surface proteins (Martinez-Nunez et al., 2010). In the alphaproteobacterium Agrobacterium tumefaciens, the TCS VirA/VirG governs the type IV secretion system that transfers oncogenic DNA into plant host cells and causes tumor formation. This pair of TCSs can sense the presence of phenolic compounds released by wounded plants, thereby triggering the activation of several genes involved in tumor induction (Lin et al., 2008). Conversely, (2H)-1,4-benzoxazin-3(4H)-one (BOA) and salicylic acid (SA), two plant phenolic compounds, can inhibit $A$. tumefaciens infection by blocking the expression of VirA/VirG (Zhang et al., 2000; Yuan et al., 2007).

The Anaplasmataceae (order Rickettsiales) encompass a group of Gram-negative bacteria with fastidious growth. They develop as obligate parasites of the vacuoles of hematopoietic cells of mammals. They have been grouped in the genera Ehrlichia, Anaplasma, and Wolbachia. Many of these species, such as Ehrlichia canis, the etiologic agent of canine monocytic ehrlichiosis (CME), E. chaffeensis, associated to human monocytic ehrlichiosis, $A$. phagocytophilum, and E. ewingii, related to human granulocytic ehrlichiosis, have significant impact on human and animal health (Rikihisa, 1991; Dumler et al., 2001). A key to the survival of these bacteria inside the vacuoles of the host cell is their capacity to resist or avoid exposition to the digestive enzymes brought to vacuoles by fusion with lysosomes (Rikihisa, 2010). Biochemical and cellular analyses of the life cycle of Anaplasmataceae have indicated that these bacteria develop mechanisms to inhibit lysosome-vacuole fusion in the host cell. Moreover, the use of closantel, an inhibitor of TCSs (Cheng et al., 2006; Kumagai et al., 2006; Rikihisa, 2010), blocks the fusion of lysosomes to the vacuoles of cells infected by $E$. chaffeensis, suggesting the involvement of the TCS in this process.

Three TCSs have been identified in the genome of E. chaffeensis and named, by analogy to their orthologs in other bacterial species, PleC/PleD, NtrY/NtrX, and CckA/CtrA. PleC, NtrY, and CckA are the genes encoding HK proteins, whereas $\mathrm{PleD}$, NtrX, and CtrA encode RR proteins (Kumagai et al., 2006). In the oligotrophic bacterium Caulobacter crescentus, CckA has been shown to be responsible for the in vivo phosphorylation of CtrA, which binds to regulatory regions of DNA and regulates the expression of several genes involved in the cell cycle (Reisenauer et al., 1999, \#1539; Jacobs et al., 2003, \#1538). This TCS is responsible for controlling several genes important in replication, segregation, DNA methylation, cell division, and morphogenesis. Also in C. crescentus, PleC/PleD is involved in control of cell differentiation (Hecht et al., 1995), 
an important stage in the life cycle of many bacteria. NtrY and NtrX have been identified and characterized in nitrogen-fixing bacteria Azorhizobium caulinodans and Azospirillum brasilense as being responsible for the regulation of genes important in nitrogen metabolism (Pawlowski et al., 1991; Ishida et al., 2002).

Doxycycline is the drug of choice for treatment of most rickettsial diseases, including CME. It provides the best results for the clinical improvement of treated animals. Nevertheless, although a series of reports confirm the elimination of E. canis in treated animals, others raise the possibility that total elimination varies with the stage of the disease (McClure et al., 2010). Therefore, new treatment regimens using new drugs, alone or in conjunction with doxycycline, are relevant for the treatment of CME and possibly other rickettsial diseases.

In this study, we performed Basic Local Alignment Search Tool analyses of the $E$. canis proteome using as a query the TCS proteins described in E. chaffeensis. We found that the same genes and their corresponding proteins are present in E. canis and other Anaplasmataceae, although their transcription activities have not yet been characterized. We present an in silico analysis of these TCS proteins and an evaluation by quantitative polymerase chain reaction (PCR) of the potential growth inhibitory activity of BOA against $E$. canis. Taken together, our results indicate that the E. canis TCS is a promising target for the development of new antiehrlichial drugs based on plant TCS inhibitors.

\section{MATERIAL AND METHODS}

\section{E. chaffeensis TCS sequences}

As query sequences for the database searches, the TCS proteins CckA (YP 507553.1), CtrA (YP_507798.1), PleC (YP_507680.1), PleD (YP_507571.1), NtrY (YP_507120.1), and NtrX (YP_507159.1) were downloaded from the GenBank protein database (Benson et al., 2011).

\section{Identification of orthologous E. chaffeensis HKs and RRs in E. canis and other Anaplasmataceae}

The proteomes of Anaplasmataceae were searched for TCS proteins using BLASTP (Altschul et al., 1997) in the Integrated Microbial Genomes (Markowitz et al., 2010) and GenBank databases with TCS protein sequences from $E$. chaffeensis as probes. Owing to high sequence identity, most top sequences showed e-values of 0.0 and therefore were considered orthologous.

\section{Sequence analysis}

The identified E. canis orthologous proteins CckA/CtrA, PleC/PleD, and NtrY/NtrX were also analyzed with the Conserved Domains Database Search Program (Marchler-Bauer et al., 2009) to identify the typical TCS domains.

\section{Strains and drug treatment}

The $E$. canis São Paulo strain (Aguiar et al., 2008) was used to infect a DH82 canine malignant histiocytic cell line (ATCC No. CRL - 10389; Wellman et al., 1988) cultured in Dulbecco's 
modified Eagle's medium supplemented with $5 \%$ bovine calf serum and incubated at $37^{\circ} \mathrm{C}$ under a $5 \% \mathrm{CO}_{2}$ atmosphere. After reaching $70-80 \%$ infection, infected cells were transferred to 24 -well plates at a concentration of $2 \times 10^{6}$ cells $/ \mathrm{mL}$ and cultured for an additional 7 days in the presence of varying concentrations of BOA (Sigma-Aldrich, USA, CAS No. 5466-88-6) and doxycycline (Sigma-Aldrich, CAS No. 24390-14-5). Substances were diluted in dimethylsulfoxide, which did not exceed $0.6 \%$ of the final concentration.

\section{Real-time PCR}

Genomic DNA from cell cultures was purified with a Genomic DNA cells and tissue kit (Macherey-Nagel) and used for real-time PCR. The single-copy GroESL gene of E. canis was selected for the development of the real-time TaqMan PCR assay. Primers that amplified a 175-bp region of the gene (bases 443 to 617, GenBank accession No. U96731) were selected. The reactions used a 1 X Master Mix (Roche Real-Time PCR Mix) in a final reaction volume of $25 \mu \mathrm{L}$ containing $200 \mathrm{nM}$ of the forward primer, ECGROTQFWD - (5'-GCGAAAGTTATAGAAGAA-3'), and reverse primer, ECGROTQREV - (5'-CACCATTAGCGGAAATAG-3'), and a $100 \mathrm{nM}$ concentration of the probe ECGROPROBE - (5'-FAM-TGAAACGTGAAATACTATCTGAAGABQH1-3'). PCR and fluorescence detection were performed using a Stratagene Mx3000P (Stratagene, La Jolla, CA, USA). The thermal cycler program was $95^{\circ} \mathrm{C}$ for $10 \mathrm{~min}$, followed by 40 cycles of $95^{\circ} \mathrm{C}$ for $15 \mathrm{~s}, 60^{\circ} \mathrm{C}$ for $20 \mathrm{~s}$, and $72^{\circ} \mathrm{C}$ for $20 \mathrm{~s}$. Standard curves were generated using a PCR fragment of GroESL with known amounts as measured with the aid of a ultraviolet spectrophotometer to calculate DNA concentration, which was converted to number of bacterial cells.

\section{RESULTS}

\section{In silico characterization of $E$. canis TCS}

In the E. canis genome, three pairs of TCS proteins were identified that can be described as orthologous to the TCS pairs CckA/CtrA, PleC/PleD, and NtrY/NtrX of E. chaffeensis. Orthologs of these sequences were also found in other species of Anaplasmataceae, and they also showed high similarity to E. chaffeensis TCS proteins (Table 1). The structural analysis of the identified TCS proteins, with aid of the CDD software, identified the characteristic HK and RR domains and revealed significant differences in the organization of these proteins (Figure 1). The CckA protein consists of 824 amino acids (aa) and shows a sensor domain and an $\mathrm{H}^{+}$-ATPase domain composed of 121 aa. Its unique characteristic (absent in the other HKs) is the presence of a phosphoacceptor domain of 64 aa, with the characteristic invariant histidine, and a receptor domain of 113 aa, with the characteristic invariant aspartate residue, in the same polypeptide chain. This type of organization distinguishes this HK as a hybrid HK and its phosphotransfer domain is known as the histidine-containing phosphotransfer domain. This type of phosphotransfer system catalyzes three reactions in which a phosphate residue is first transferred from the phosphoacceptor domain to the phosphoreceptor domain. A second reaction occurs through transfer of the phosphate residue from the phosphoreceptor domain to the histidine-containing phosphotransfer domain. Finally, the phosphate is transferred to the receptor domain in the RR. This additional step of phosphorylation may allow bacteria to integrate multiple signal "inputs" into a single-signal output or signal leading to transcriptional 
regulation (Stock et al., 2000). The CtrA protein is the corresponding RR of CckA and can be considered a typical RR. It contains 265 aa, a receptor domain composed of 115 aa, and a transcriptional regulator composed of 95 aa.

\begin{tabular}{|c|c|c|c|c|c|c|}
\hline \multirow[b]{3}{*}{ Ehrlichia canis Jake } & \multicolumn{6}{|c|}{ Ehrlichia canis Jake } \\
\hline & \multicolumn{3}{|c|}{ Sensor kinase } & \multicolumn{3}{|c|}{ Response regulator } \\
\hline & CckA YP_302961 & PleC YP_302862 & NtrY YP_303334 & CtrA YP_303446 & PleD YP_302946 & NtrX YP_303298 \\
\hline $\begin{array}{l}\text { Ehrlichia chaffeensis } \\
\text { str. Arkansas }\end{array}$ & YP_50755̄3 89\% & YP_507680 83\% & YP_507120 78\% & YP_5077̄̄98 96\% & YP_5075̄1 94\% & YP_507159 92\% \\
\hline $\begin{array}{l}\text { Ehrlichia ruminantium } \\
\text { Welgevonden }\end{array}$ & YP_180200 74\% & YP_180081 76\% & YP_180559 75\% & YP_180649 90\% & YP_180185 84\% & YP_197569 91\% \\
\hline $\begin{array}{l}\text { Ehrlichia ruminantium } \\
\text { Gardel }\end{array}$ & YP_196265 75\% & YP_196135 75\% & YP_196649 75\% & YP_196747 90\% & YP_196249 84\% & YP_196610 91\% \\
\hline $\begin{array}{l}\text { Anaplasma marginale } \\
\text { St. Marie }\end{array}$ & YP_153783 57\% & YP_153666 52\% & YP_153544 42\% & YP_154154 96\% & YP_153767 67\% & YP_153584 71\% \\
\hline
\end{tabular}

NtrY

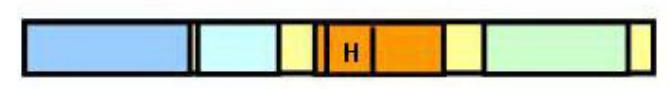

PleC

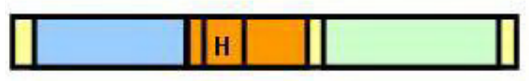

CckA

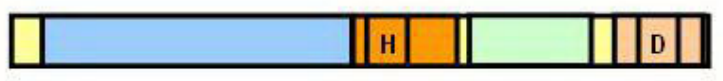

NtrX

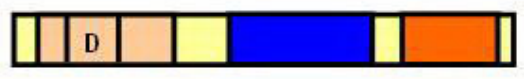

PleD

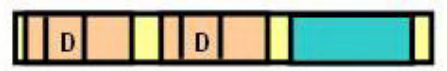

CtrA
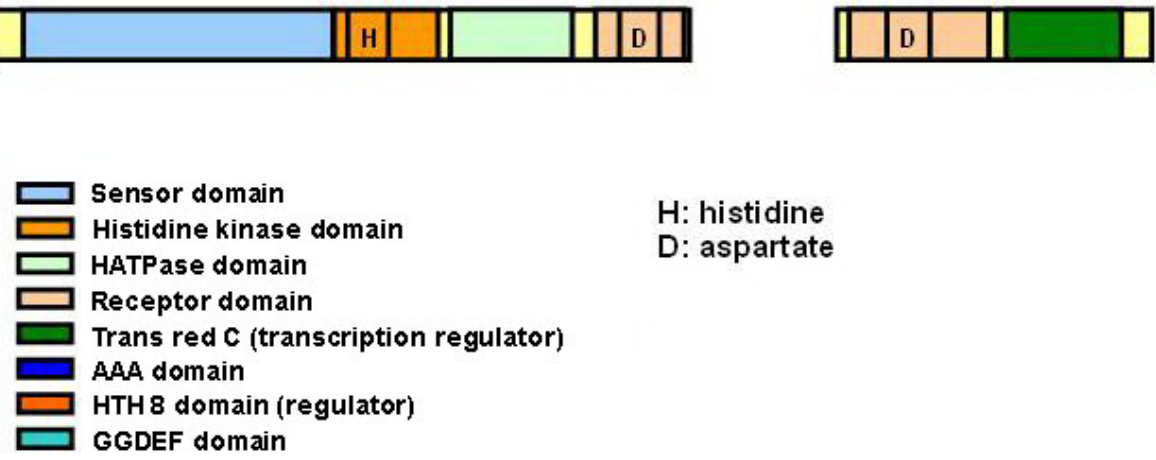

H: histidine

D: aspartate

Figure 1. Domains of two-component system proteins identified with aid of the Conserved Domains software.

NtrY contains 713 aa and has a sensor domain, a histidine kinase, adenylyl cyclase, methyl-accepting protein, and phosphatase (HAMP) domain, and a phosphoacceptor $\mathrm{H}^{+}$ATPase domain. The HAMP domain is an interlinker region that connects the extracellular sensory and intracellular signaling domains of the HKs (Hulko et al., 2006). NtrX protein, the 
corresponding RR, consists of 471 aa and has three domains, a receptor domain composed of 114 aa, an AAA (ATPase associated with cellular activities) domain comprising 204 aa, and an HTH domain, which is involved in DNA binding for transcriptional regulation.

The PleC protein has 470 aa and is a typical HK, formed by a field sensor, a phosphoacceptor domain composed of 64 aa, and an $\mathrm{H}^{+}$-ATPase domain of 105 aa. The PleD is an RR with 458 aa. It features two receiver domains with a characteristic invariant aspartate residue rather than a normal one. It also displays the domain $\mathrm{GG}(\mathrm{D} / \mathrm{E})$ or $\mathrm{EF}$, which is involved in the synthesis and hydrolysis of cyclic guanosine monophosphate, a compound that acts as second messenger in various bacterial species (Ryjenkov et al., 2005).

\section{Evaluation of BOA inhibition of $E$. canis growth using quantitative PCR}

In the experimental conditions described above, the quantitative PCR assay indicated a low inhibitory effect of BOA on E. canis growth. Inhibition started at $9.0 \%$ at a concentration of $62.5 \mu \mathrm{g} / \mathrm{mL}$ and reached $12.1 \%$ at $500 \mu \mathrm{g} / \mathrm{mL}$. Doxycycline, used as a control at a $4 \mu \mathrm{g} / \mathrm{mL}$, had an inhibitory effect of $16.9 \%$ (Table 2 ).

Table 2. Inhibitory effect of (2H)-1,4-benzoxazin-3(4H)-one (BOA) on Ehrlichia canis growth evaluated by qPCR.

\begin{tabular}{lcc}
\hline Substance & Concentration $(\mu \mathrm{g} / \mathrm{mL})$ & Inhibition $(\%)$ \\
\hline Doxycycline & 4 & $16.9 \pm 3.30$ \\
BOA & 62.5 & $9.0 \pm 3.54$ \\
BOA & 125 & $9.3 \pm 2.59$ \\
BOA & 250 & $11.8 \pm 2.73$ \\
BOA & 500 & $12.1 \pm 3.41$ \\
\hline
\end{tabular}

\section{DISCUSSION}

The basis of antibacterial drug research has been a whole-cell antibacterial approach seeking molecules that mostly destroy bacterial structure. With the rise of genomics, new strategies for finding molecules that are inhibitors of more complex processes of bacterial pathogenesis have become feasible (Finlay, 2010). In this regard, natural products continue to be an enormous source of bioactive compounds with inhibitory activity against microorganisms. Recently, SA and BOA, two plant phenolic metabolites that inhibit bacterial TCSs, have been described as potential leading molecules for use as herbicides against phytopathogens. The high degree of conservation of TCS HK and RR proteins, in view of their active sites, indicates that SA and BOA could be explored in the development of drugs inhibiting this class of proteins with great potential to inhibit several TCSs in various bacterial species. In this study, the three pairs of TCS genes previously identified in E. chaffeensis - CckA/CtrA, PleC/ $\mathrm{PleD}$, and NtrY/NtrX - were identified in E. canis and other Anaplasmataceae species through in silico analysis of their proteomes.

This analysis demonstrated a high similarity between proteins encoded by the TCS genes of the genus Ehrlichia, as well those of other Anaplasmataceae. On average, the similarity was $60.1 \%$ for the HK proteins and $76.6 \%$ for the RR proteins. It was particularly high in the phosphoacceptor and receiver domains of the HK and RR proteins, respectively. Despite being present in almost all bacterial species, TCSs are absent in mammals (Gotoh et al., 2010), and in 
the case of Ehrlichia sp, this absence is an interesting feature because this group of endobacteria infects the cells of mammalian species, including humans. Therefore, as in other bacteria, the TCS in these organisms is an interesting target for the development of antiehrlichial drugs that could also be used against other species of the Anaplasmataceae family. The study of E. canis inhibition by BOA showed low activity, but it suggests that new experiments should be performed using derivatives of these metabolites and other phenolic compounds such as SA. These new drugs could be used in conjunction with doxycycline and enhance the effectiveness of CME treatment.

\section{ACKNOWLEDGMENTS}

Research supported by grants from Fundação de Amparo à Pesquisa do Estado de São Paulo (\#07/07234-6, \#2008/04778-8, \#2008/05011-2, \#2008/08146-6) and Coordenação de Aperfeiçoamento de Pessoal de Nível Superior/Programa de Suporte à Pós-Graduação de Instituições de Ensino Particulares. We thank the staff of the Biotechnology Unit, Universidade de Ribeirão Preto, for general support and K.M. for English revision.

\section{REFERENCES}

Aguiar DM, Hagiwara MK and Labruna MB (2008). In vitro isolation and molecular characterization of an Ehrlichia canis strain from São Paulo, Brazil. Braz. J. Microbiol. 39: 489-493.

Altschul SF, Madden TL, Schaffer AA, Zhang J, et al. (1997). Gapped BLAST and PSI-BLAST: a new generation of protein database search programs. Nucleic Acids Res. 25: 3389-3402.

Armitage JP (1992). Behavioral responses in bacteria. Annu. Rev. Physiol. 54: 683-714.

Benson DA, Karsch-Mizrachi I, Lipman DJ, Ostell J, et al. (2011). GenBank. Nucleic Acids Res. 39: D32-D37.

Cheng Z, Kumagai Y, Lin M, Zhang C, et al. (2006). Intra-leukocyte expression of two-component systems in Ehrlichia chaffeensis and Anaplasma phagocytophilum and effects of the histidine kinase inhibitor closantel. Cell Microbiol. 8: $1241-1252$.

Dumler JS, Barbet AF, Bekker CP, Dasch GA, et al. (2001). Reorganization of genera in the families Rickettsiaceae and Anaplasmataceae in the order Rickettsiales: unification of some species of Ehrlichia with Anaplasma, Cowdria with Ehrlichia and Ehrlichia with Neorickettsia, descriptions of six new species combinations and designation of Ehrlichia equi and "HGE agent" as subjective synonyms of Ehrlichia phagocytophila. Int. J. Syst. Evol. Microbiol. 51: 2145-2165.

Finlay BB (2010). The art of bacterial warfare. Sci. Am. 302: 56-63.

Gao R and Stock AM (2009). Biological insights from structures of two-component proteins. Ann. Rev. Microbiol. 63: 133-154.

Gotoh Y, Eguchi Y, Watanabe T, Okamoto S, et al. (2010). Two-component signal transduction as potential drug targets in pathogenic bacteria. Curr. Opin. Microbiol. 13: 232-239.

Hecht GB, Lane T, Ohta N, Sommer JM, et al. (1995). An essential single domain response regulator required for normal cell division and differentiation in Caulobacter crescentus. EMBO J. 14: 3915-3924.

Hulko M, Berndt F, Gruber M, Linder JU, et al. (2006). The HAMP domain structure implies helix rotation in transmembrane signaling. Cell 126: 929-940.

Ishida ML, Assumpcao MC, Machado HB, Benelli EM, et al. (2002). Identification and characterization of the twocomponent NtrY/NtrX regulatory system in Azospirillum brasilense. Braz. J. Med. Biol. Res. 35: 651-661.

Jacobs C, Ausmees N, Cordwell SJ, Shapiro L, et al. (2003). Functions of the CckA histidine kinase in Caulobacter cell cycle control. Mol. Microbiol. 47: 1279-1290.

Kumagai Y, Cheng Z, Lin M and Rikihisa Y (2006). Biochemical activities of three pairs of Ehrlichia chaffeensis twocomponent regulatory system proteins involved in inhibition of lysosomal fusion. Infect. Immun. 74: 5014-5022.

Laub MT and Goulian M (2007). Specificity in two-component signal transduction pathways. Annu. Rev. Genet. 41: 121-145.

Lavin JL, Kiil K, Resano O, Ussery DW, et al. (2007). Comparative genomic analysis of two-component regulatory proteins in Pseudomonas syringae. BMC Genomics 8: 397.

Lin YH, Gao R, Binns AN and Lynn DG (2008). Capturing the VirA/VirG TCS of Agrobacterium tumefaciens. Adv. Exp. 
Med. Biol. 631: 161-177.

Marchler-Bauer A, Anderson JB, Chitsaz F, Derbyshire MK, et al. (2009). CDD: specific functional annotation with the conserved domain database. Nucleic Acids Res. 37: D205-D210.

Markowitz VM, Chen IM, Palaniappan K, Chu K, et al. (2010). The integrated microbial genomes system: an expanding comparative analysis resource. Nucleic Acids Res. 38: D382-D390.

Martinez-Nunez C, Altamirano-Silva P, Alvarado-Guillen F, Moreno E, et al. (2010). The two-component system BvrR/ BvrS regulates the expression of the type IV secretion system VirB in Brucella abortus. J. Bacteriol. 192: 5603-5608.

McClure JC, Crothers ML, Schaefer JJ, Stanley PD, et al. (2010). Efficacy of a doxycycline treatment regimen initiated during three different phases of experimental ehrlichiosis. Antimicrob. Agents Chemother. 54: 5012-5020.

Miller LD, Russell MH and Alexandre G (2009). Diversity in bacterial chemotactic responses and niche adaptation. $A d v$. Appl. Microbiol. 66: 53-75.

Pawlowski K, Klosse U and de Bruijn FJ (1991). Characterization of a novel Azorhizobium caulinodans ORS571 twocomponent regulatory system, NtrY/NtrX, involved in nitrogen fixation and metabolism. Mol. Gen. Genet. 231: 124-138.

Reisenauer A, Quon K and Shapiro L (1999). The CtrA response regulator mediates temporal control of gene expression during the Caulobacter cell cycle. J. Bacteriol. 181: 2430-2439.

Rikihisa Y (1991). The tribe Ehrlichieae and ehrlichial diseases. Clin. Microbiol. Rev. 4: 286-308.

Rikihisa Y (2010). Molecular events involved in cellular invasion by Ehrlichia chaffeensis and Anaplasma phagocytophilum. Vet. Parasitol. 167: 155-166.

Ryjenkov DA, Tarutina M, Moskvin OV and Gomelsky M (2005). Cyclic diguanylate is a ubiquitous signaling molecule in bacteria: insights into biochemistry of the GGDEF protein domain. J. Bacteriol. 187: 1792-1798.

Stock AM, Robinson VL and Goudreau PN (2000). Two-component signal transduction. Annu. Rev. Biochem. 69: 183-215.

Wellman ML, Krakowka S, Jacobs RM and Kociba GJ (1988). A macrophage-monocyte cell line from a dog with malignant histiocytosis. In Vitro Cell Dev. Biol. 24: 223-229.

Yuan ZC, Edlind MP, Liu P, Saenkham P, et al. (2007). The plant signal salicylic acid shuts down expression of the vir regulon and activates quormone-quenching genes in Agrobacterium. Proc. Natl. Acad. Sci. U. S. A. 104: 11790-11795.

Zhang J, Boone L, Kocz R, Zhang C, et al. (2000). At the maize/Agrobacterium interface: natural factors limiting host transformation. Chem. Biol. 7: 611-621. 